\title{
Sexual health issues in adolescents and young adults
}

Authors: Sophie Forsyth ${ }^{A}$ and Karen Rogstad $B$

Authors: Aconsultant, Great Western Hospitals NHS Foundation Trust, Swindon, UK; Bconsultant in HIV and sexual health and undergraduate sub-dean, Sheffield Teaching Hospitals NHS Foundation Trust; on behalf of the BASHH Adolescent Special Interest Group

Clinical Medicine 2015;15:447-51.

The affiliations were listed in the incorrect order. The correct affiliation list is printed above.

\section{Tackling emerging infections: clinical and public health lessons from the West African Ebola virus disease outbreak, 2014-2015}

\section{Authors: Naomi F Walker ${ }^{A}$ and Christopher JM WhittyB}

Authors: Aspecialist trainee in tropical and general internal medicine, Clinical Research Department, London School of Hygiene and Tropical Medicine, London, UK; B professor of public and international health, Department of Infectious Diseases and Immunity, Imperial College London, London, UK, and Clinical Infectious Diseases Research Initiative, Institute of Infectious Disease and Molecular Medicine, University of Cape Town, Cape Town, South Africa

Clinical Medicine 2015;15:457-60.

The affiliations were listed in the incorrect order. The correct affiliation list is printed above. 